\title{
Effects of salt marsh restoration on eukaryotic microbenthic communities in the Yangtze Estuary
}

\author{
Y. Xu ${ }^{1}$, S. Yao ${ }^{2}$, K. Soetaert ${ }^{3}$, X. Fan ${ }^{2, *}$ \\ ${ }^{1}$ State Key Laboratory of Estuarine and Coastal Research, East China Normal University, 200241 Shanghai, PR China \\ ${ }^{2}$ School of Life Sciences, East China Normal University, 200241 Shanghai, PR China \\ ${ }^{3}$ Department of Estuarine and Delta Systems, NIOZ Royal Netherlands Institute for Sea Research, and Utrecht University, \\ PO Box 140, 4400AC Yerseke, The Netherlands
}

\begin{abstract}
To fight invasion of Spartina alterniflora and conserve biodiversity of migratory shorebirds, several $S$. alterniflora marshes and bare mudflats have been restored back to Scirpus mariqueter marshes in the Yangtze River estuary in recent years. Although it is known that changes in plant cover in salt marshes alters the physical environment, findings on its effects on benthic communities are inconsistent and site-specific. In the present study, we tested how ciliates were influenced by a recent restoration. Ciliated protozoa play a key role in wetland benthic food webs and are useful bioindicators. We investigated 3 habitats: restored $S$. mariqueter meadow, $S$. alterniflora-invaded meadow, and bare mudflats, where we quantified environmental conditions, community composition (species and traits), and 3 aspects of biodiversity (classic, taxonomic, and functional diversity) over 4 seasons. CAP and ANOSIM were used to determine the differences among samples, while RDA and GLMs were used to reveal potential driving factors. We found that, although changes in the type of vegetation due to restoration or invasion significantly modified the sediment properties and ciliate species composition, it had limited influence on functional trait composition as well as on diversity, especially on functional diversity. These results indicate that the conversion of mudflats to $S$. mariqueter meadow did not affect ciliate community functions at the time scale of this study. However, biomonitoring based on microbenthic communities should be included when evaluating the long-term success of coastal wetland restoration.
\end{abstract}

KEY WORDS: Ciliates · Functional diversity · Trait composition · Wetland · Exotic plant

\section{INTRODUCTION}

Coastal salt marshes provide important ecosystem services. One of their essential functions is conserving high biodiversity of native species, including nekton, invertebrates, waterbirds, and cryptic microorganisms, through providing food sources and diverse habitats (Ferguson \& Rakocinski 2008, X. Li et al. 2018). However, salt marsh ecosystems are facing great pressures due to anthropogenic activities, such as reclamation, eutrophication, exotic species invasion, and habitat alteration, including restoration, which could fundamentally change the physical pro-

${ }^{*}$ Corresponding author: xpfan@bio.ecnu.edu.cn

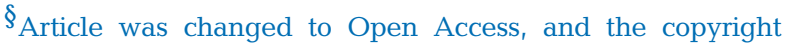
notice updated after publication.

This corrected version: April 15, 2020 perties and ecological dynamics of marshes (Gedan et al. 2009, X. Li et al. 2018).

Salt marshes in the Yangtze River estuary are undergoing rapid environmental changes. In particular, the changes resulting from the invasion of Spartina alterniflora have received considerable attention over the last 2 decades (Chen et al. 2007, Li et al. 2009, Wang et al. 2010, Quan et al. 2016). S. alterniflora was first introduced into the Yangtze River estuary in the late 1990s for coastal protection and sediment trapping, but then spread rapidly along the east coast of China (Li et al. 2009, Quan et al. 2016). In 2005, S. alterniflora marshes accounted for $49.4 \%$ of the total

( ) The authors 2020. Open Access under Creative Commons by Attribution Licence. Use, distribution and reproduction are unrestricted. Authors and original publication must be credited.

Publisher: Inter-Research · www.int-res.com 
marsh area in the Dongtan wetland in this estuary (Li et al. 2009). Its fast expansion negatively impacted native plants, including Scirpus mariqueter, which is endemic to China and mostly distributed in the Yangtze River estuary (He et al. 2012). Since S. mariqueter marshes are a key habitat to waterbirds and migrants, the disappearance of $S$. mariqueter has a great influence on bird communities (Gan et al. 2009).

As the Yangtze River estuary is an important stopover site for the migratory shorebirds in the East Asian-Australasian Flyway, one of its valuable functions is conserving bird diversity, including some rare and endangered species (Barter et al. 1997, Gan et al. 2009). Therefore, in recent years, restoration through converting $S$. alterniflora stands and bare mudflats into $S$. mariqueter marshes has been implemented. Both natural and human-mediated changes in plant cover influence the hydrodynamics and deposition of sediments, and therefore alter the physical environment and ecosystem processes, e.g. nutrient cycling and carbon allocation, which relate tightly to the structure of associated animal communities (Cheng et al. 2006, Whitcraft \& Levin 2007, C. Li et al. 2018). Therefore, it is important to evaluate not only vegetative characteristics, but also the impacts on benthic communities from restoration in order to minimize its current and future influences on the benthic ecosystem (Alphin \& Posey 2000, Huspeni \& Lafferty 2004). However, few studies have been carried out on the effects of the changes caused by restoration on microhabitats, and available studies on benthic communities have primarily focused on the macrobenthos (Alphin \& Posey 2000, Moseman et al. 2004, Ferguson \& Rakocinski 2008).

Although eukaryotic microbial organisms play key roles in wetland benthic food webs, since they predate bacteria, benthic algae, and organic particles, they have been largely ignored in biomonitoring programs due to the difficulties in identifying them (Hamels et al. 2005, Weisse 2006, Weisse \& Sonntag 2016, Cordier et al. 2018). As single-celled organisms, they are highly sensitive to environmental changes, especially impacts caused by human activities such as organic enrichment and heavy metal contaminations (Madoni 2000, Stoeck et al. 2018). Due to their short generation times, protists have the ability to adapt much faster after environmental changes compared with macrofauna, which have much longer generation times. Craft \& Sacco (2003) suggested that, as some groups of macrofauna may need as much as $25 \mathrm{yr}$ to achieve equilibrium, monitoring after salt marsh construction should be carried out for longer than $5 \mathrm{yr}$. Therefore, employing single-celled eukaryotes as bioindicators could easily provide an early warning system for ecosystem dysfunction in marshes undergoing restoration, followed by longer-term assessment of other parameters. Among eukaryotes, ciliates have been successfully used to assess environmental status in many coastal environments, including aquaculture waters and estuary wetlands (Xu et al. 2016, 2018, Forster et al. 2019). Ciliated protozoa are more easily characterized taxonomically and functionally compared with other groups of protozoa, which makes them suitable for use in monitoring programs.

While previous studies mainly included classic descriptors such as abundance (individual number), few have also incorporated taxonomic and functional diversity (Alphin \& Posey 2000, Ferguson \& Rakocinski 2008). It has been demonstrated that since species replacements do not always lead to changes in ecological functioning, classical diversity based on species identity and abundance provide an incomplete view of biodiversity, as they do not take into account the taxonomic and functional differences among species (Bremner et al. 2003, Villéger et al. 2010). Therefore, an integrative approach combining the 3 aspects of biodiversity, i.e. classical, taxonomic, and functional diversity, is more informative and can provide a better understanding of general relationships between community structure and ecosystem functions (McGill et al. 2006, Villéger et al. 2008, 2011, Gusmao et al. 2016).

The rapid change in the dominant species of vegetation caused by salt marsh restoration and S. alterniflora invasions in the Yangtze River estuary in eastern China provide a good opportunity to study their influences on the benthic ecosystem. In the present study, we compared benthic ciliate species and trait composition, as well as 3 aspects of biodiversity of newly created $S$. mariqueter marshes, invaded marshes, and bare mudflats over 4 seasons. Our aim was to address the following questions. (1) How does benthic ciliate community composition vary among the 3 habitats, at the taxonomic and trait level, and what are the main driving factors behind their variation? (2) Does the restoration of native $S$. mariqueter marshes from bare mudflats have significant impacts on benthic ciliate community functions?

\section{MATERIALS AND METHODS}

\subsection{Study sites and sampling}

The study was carried out in the tidal marshes of the Nanhui wetland in the Yangtze River es- 
tuary, China $\left(30^{\circ} 57^{\prime} 38^{\prime \prime}-30^{\circ} 52^{\prime} 34^{\prime \prime} \mathrm{N}, 121^{\circ} 55^{\prime} 0^{\prime \prime}-\right.$ $121^{\circ} 57^{\prime} 27^{\prime \prime} E_{i}$ Fig. 1). This wetland area was created after a reclamation project in 2000-2002. Spartina alterniflora (hereinafter called Spartina) first spread to the newly formed bare mudflats from other wetlands in the Yangtze Estuary and created dense and pure marshes in the east and west ends of this area (Fig. 1). In order to prevent further spreading of Spartina and establish beneficial habitat for migratory waterbirds, native Scirpus mariqueter (hereinafter called Scirpus) was planted in the middle bare mudflats in 2015 (C. Li et al. 2018). After about 3 yr, the re-built Scirpus marsh had formed a dense stand about 1 $\mathrm{km}$ long along the dyke and $300 \mathrm{~m}$ wide towards the sea (Fig. 1). This Scirpus marsh is mixed with Spartina in some areas close to the dyke, while in the lower tidal zone they form monocultures (Fig. 1).

Sampling was carried out in April (spring), July (summer), October (autumn), and December (winter) 2018. All samples were taken at low tide, and during the sampling the sediment remained moist. Three sampling sites in 3 habitats were selected, i.e. created Scirpus marshes, Spartina marshes, and bare mudflats in the middle intertidal zone (Fig. 1). The 3 sites of the Spartina marshes and bare mudflats are separated by spur dykes, while those of the Scirpus marshes are separated by tidal creeks (Fig. 1B,H). In the middle of each sampling site, three replicate $1 \mathrm{~m}^{2}$ sampling plots along the transect parallel to the coastline were selected haphazardly which were spaced about $10 \mathrm{~m}$ apart (108 samples in total). Within each plot, the upper $2 \mathrm{~cm}$ of the sediment was collected 3 times randomly using a modified syringe $\left(2.2 \mathrm{~cm}\right.$ in diameter, ca. $4 \mathrm{~cm}^{2}$ in area) and then mixed with glutaraldehyde $(2 \%$ final concentration) (Lei \& Xu 2011); 3 additional sediment samples were randomly collected from the top $2 \mathrm{~cm}$ of each plot and then pooled together for measuring environmental variables. For nutrient analyses, a water sample overlying the sediment was taken using Millipore syringe filters with a pore size of $0.45 \mu \mathrm{m}$, and then $1.5 \% \mathrm{v} / \mathrm{v}$ saturated $\mathrm{HgCl}_{2}$ added. A quadrat frame $(0.5 \times$ $0.5 \mathrm{~m}$ ) was used to measure the height of the vegetation.

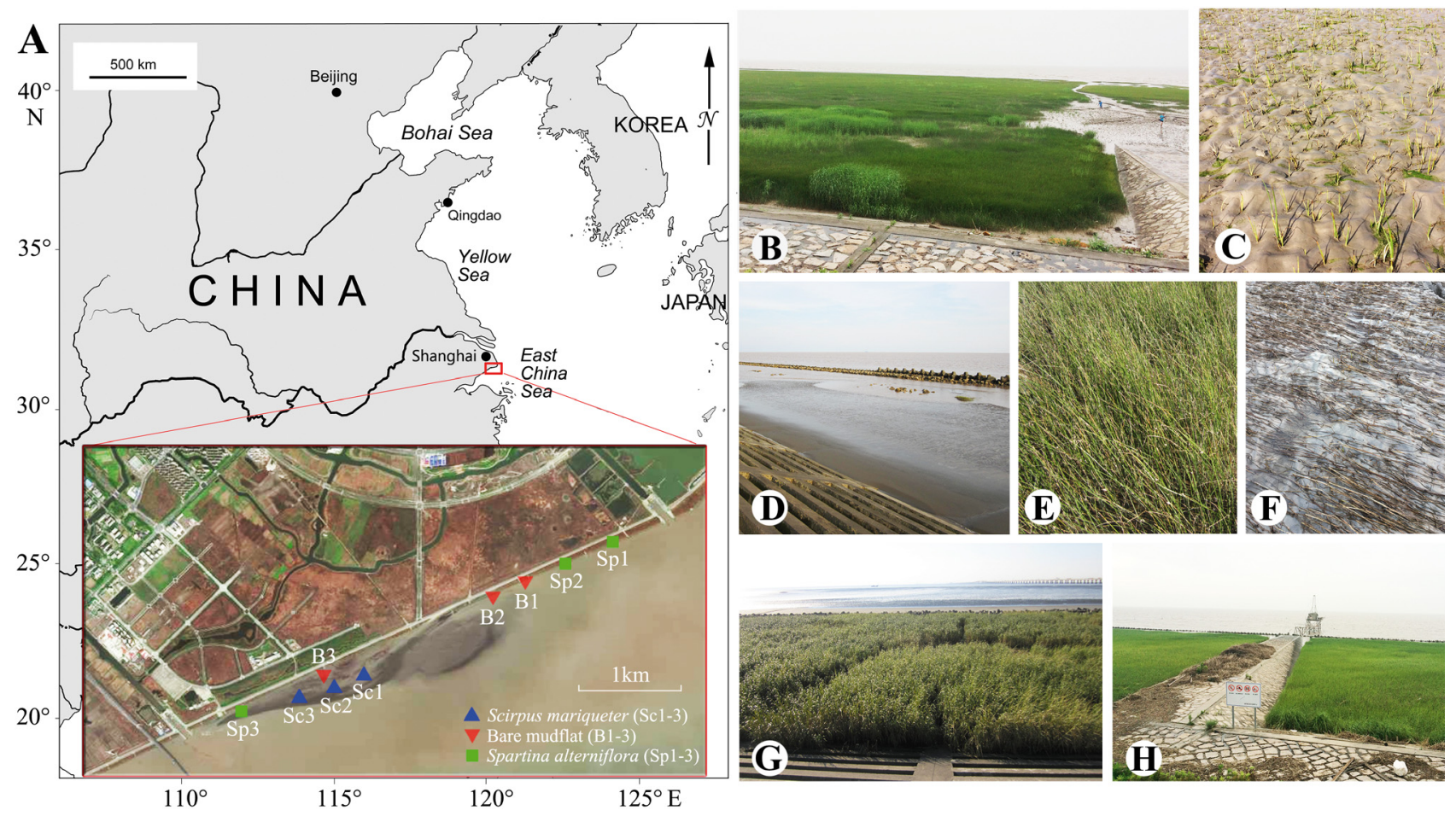

Fig. 1. (A) The 9 sampling sites in 3 habitats (Spartina alterniflora-invaded marshes, established Scirpus mariqueter marshes, and bare mudflats) in Nanhui coastal wetland, Yangtze Estuary, China. (B) S. mariqueter marshes in July. (C) Detailed view of S. mariqueter marshes in April. (D) Bare mudflat. (E) Detailed view of $S$. mariqueter marshes in October. (F) $S$. mariqueter marshes in December. (G) Detailed view of $S$. alterniflora marshes in October. (H) Sites Sp1 and Sp2 in S. alterniflora marshes, separated by spur dyke 


\subsection{Ciliate identification and trait analysis}

Ciliates were extracted and stained from all 108 samples according to $\mathrm{Xu}$ et al. (2010). At the end of this quantitative protargol stain (QPS) method, in total 216 permanent slides containing extracted and stained specimens from each sample were created. Enumeration and identification was conducted using microscopy (Olympus BX53) at 200× to $1000 \times$ magnifications. Each species was photographed at $400 \times$ or 1000× magnification. All electronic photos and permanent slides were deposited in the Laboratory of Protozoology, East China Normal University (available from the first author by request). Identifications followed mainly Carey (1992) and Lynn \& Small (2002). For each taxon, cell lengths of at least 5 individuals (or all individuals encountered if fewer than 5 were found) were measured with an ocular micrometer. The species abundance dataset was reduced to retain only those taxa found in at least 2 samples to avoid influence of too-rare species.

Five traits, sub-divided into 13 categories, were selected according to $\mathrm{Xu} \&$ Soininen (2019). The traits reflect morphological characteristics (body size, degree of flexibility, and body form) and behavior (feeding and mobility) (Table S1 in the Supplement at www.int-res.com/articles/suppl/m638p039_supp. xls). Data on traits were mainly obtained from the literature (Pratt \& Cairns 1985, Lynn 2008). A fuzzycoding procedure was used to account for an individual taxon displaying multiple trait categories according to $\mathrm{Xu}$ et al. (2018). After obtaining the 'taxa abundance by samples' and 'taxa by traits' data matrices, we then calculated the 'traits by samples' matrix using the 'FD' package in R 3.5.3 (Lepš et al. 2011, Laliberté et al. 2014). Analysis of 'traits by samples' showed how samples varied in terms of their functional trait composition.

\subsection{Measurement of environmental parameters}

Salinity, pH, and temperature of the water overlying the sediment were measured in situ at each sampling plot using a salinometer and a pH meter (Spectrum, pH400). Sediment grain size (SED) was determined with a laser particle-size analyzer (Beckman Coulter LS13 320). Total organic carbon (TOC) and total nitrogen (TN) contents were determined using a Vario EL CHNOS elemental analyzer (as \% dry weight of the sediment). Nutrients (ammonia, nitrate, nitrite, phosphate, and silicate) were determined using a segmented flow analyzer (SKALAR Sanplus System).

\subsection{Diversity indices}

Three classic diversity variables, i.e. total number of species $(S)$, total number of individuals $(\mathrm{N})$, Margalef's richness $(d)$, and a taxonomic diversity index (average taxonomic distinctness, AvTD) were calculated using the submodule DIVERSE in the PRIMER v.7.0.11 package (Warwick \& Clarke 1995, Clarke et al. 2014). An index of functional diversity, functional divergence (FDiv), was computed using the 'FD' package in R 3.5.3 (Laliberté et al. 2014).

\subsection{Data analysis}

Abundance data were square-root transformed prior to analysis in order to downweigh the contributions of quantitatively dominant species to the BrayCurtis similarity which do not incorporate any scaling of each species by its total or maximum across all samples (Clarke et al. 2014). Environmental variables, except for $\mathrm{pH}$ and temperature, were logtransformed to remove the right-skewness and then normalized to put them onto a common scale. The Bray-Curtis similarity matrix based on species data and a Euclidean distance matrix based on environmental variables were used in the submodule CAP (canonical analysis of principal coordinates) of PERMANOVA to show differences among communities (Anderson et al. 2008). Gowdis distance matrices were generated based on traits by samples data and log-transformed diversity indices. Three-way ANOSIMs were used to determine significance of differences of environmental conditions and community structure between samples with a mixed nested and crossed design: 'sampling site' as a nested factor in 'habitat' but crossed with 'season' (Clarke et al. 2014). In order to reveal seasonal changing patterns in community composition and environmental conditions, we used RELATE to test the significance of agreement between community composition matrices/environmental matrix and a cycle model matrix within each habitat. RELATE calculates rank correlations between the elements of 2 similarity/distance matrices and produces a matching coefficient $(\rho)$, which is then used in a permutation test (Clarke \& Ainsworth 1993). CAP, ANOSIM, and RELATE were performed in PRIMER 7+ PERMANOVA (PRIMERE). We then used redundancy analysis (RDA) to explain the main drivers in benthic ciliate species and traits composition. RDA was applied with forward selection, using Monte Carlo permutation tests (999 permutations), to select only those variables that 
significantly explained variation in the benthic ciliate communities. Finally, we used a generalized linear model (GLM) with Gaussian type on diversity indices to examine the potential drivers. We started with a full model, and the most parsimonious model was selected based on Akaike's information criterion (AIC). Forward selection, RDA, GLM, and AIC were carried out using the 'packfor', 'vegan', and 'MASS' packages in R 3.5.3 (R Development Core Team 2018).

\section{RESULTS}

\subsection{Environmental variables}

The ranges of all environmental variables in the 3 habitats in the 4 seasons are summarized in Fig. 2 and Table 1. Sediments were coarser in the bare flats, with median grain size varying between 39 and $65 \mu \mathrm{m}$, compared to the marsh sites, where SED was mostly $<30 \mu \mathrm{m}$. TOC content $(0.076-0.32 \%)$ was higher in Spartina marshes than in bare flats and Scirpus stands, except for in October, when the highest value was found in the bare flats. TN content $(0.022-0.058 \%)$ was relatively similar in all sites. In general, nitrate concentration in the overlying water (3.6-82.3 $\mu \mathrm{mol} \mathrm{l}^{-1}$ ) tended to be higher than the ammonium concentration (3.9-31.3 $\left.\mu \mathrm{mol} \mathrm{l}^{-1}\right)$ and slightly higher in the bare mudflats compared to the 2 marsh sites. Phosphate and silicate concentrations were significantly lower in April $\left(<1 \mu \mathrm{mol} \mathrm{l}^{-1}\right.$ and $7-20.8 \mu_{\mathrm{mol} \mathrm{l}}^{-1}$ for phosphate and silicate respectively) compared to the other months (1.4-2.8 and 46.5-122 $\mathrm{mol} \mathrm{l}^{-1}$ respectively) for all sites. Nitrite concentration (0.1-1.1 $\mu \mathrm{mol} \mathrm{l^{-1 }}$ ) was higher in July compared to the other months. Temperature varied from $2.1^{\circ} \mathrm{C}$ in December to $28.3^{\circ} \mathrm{C}$ in July, while salinity ranged between 8 and 17.2. $\mathrm{pH}$ varied between 7.2 and 9.2 and was generally similar in all sites. Spartina was conspicuously larger $(34-121 \mathrm{~cm})$ than Scirpus (9-34 cm).

The CAP clearly separated the 3 habitats along the second canonical axis (CAP2) and the 4 seasons
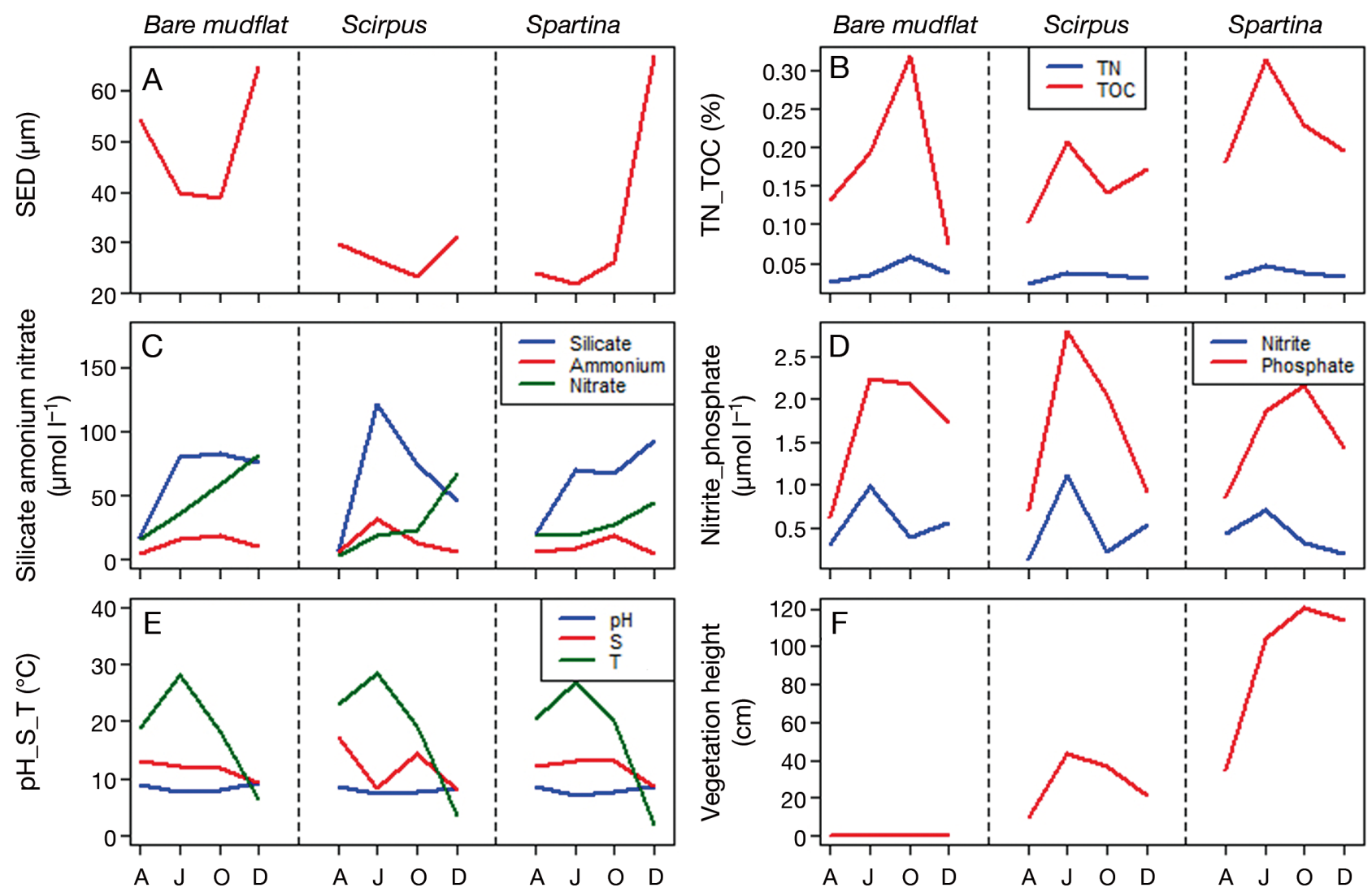

Fig. 2. Variation in environmental factors among the 3 habitats and 4 seasons (A: April; J: July; O: October; D: December). (A) Sediment grain size (SED); (B) total nitrogen (TN) and total organic carbon (TOC); (C) silicate, nitrate, and ammonium; (D) nitrite and phosphate; $(\mathrm{E}) \mathrm{pH}$, salinity $(\mathrm{S})$, and temperature $(\mathrm{T})$; and $(\mathrm{F})$ vegetation height 


\begin{tabular}{|c|c|c|c|}
\hline 买氖 & $\begin{array}{llll}0 & 0 & 0 & 0 \\
+1 & +1 & +1 & +1 \\
0 & 0 & 0 & 0\end{array}$ & 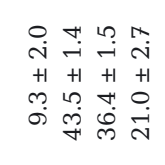 & 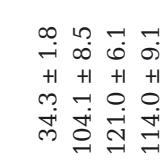 \\
\hline$H \underbrace{\tilde{U}}_{0}$ & 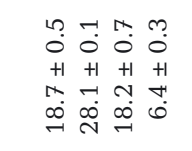 & 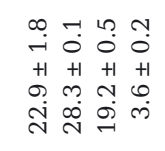 & 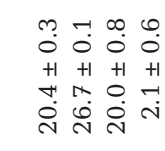 \\
\hline is & 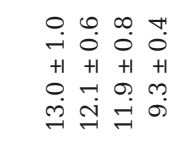 & 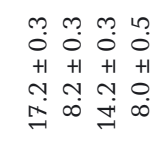 & 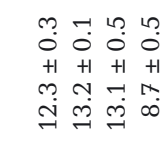 \\
\hline 窝 & $\begin{array}{llll}\rightarrow-1 & -1 & 0 & 0 \\
0 & 0 & 0 & 0 \\
+1 & +1 & +1 & +1 \\
0 & 0 & 0 & \sim \\
\infty & 0 & \infty & 0\end{array}$ & 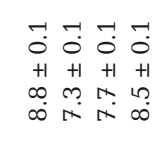 & 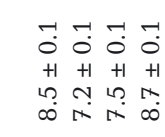 \\
\hline 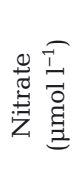 & 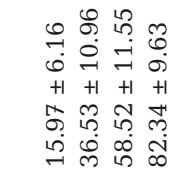 & 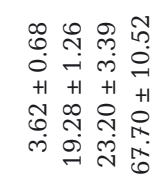 & 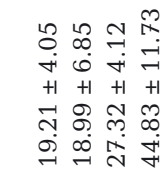 \\
\hline 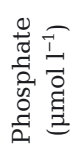 & 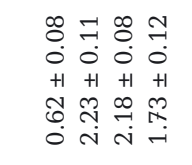 & 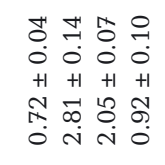 & 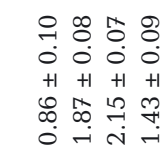 \\
\hline 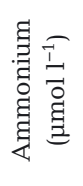 & 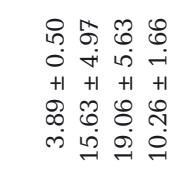 & 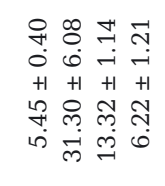 & 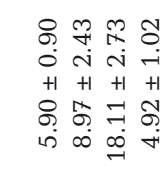 \\
\hline 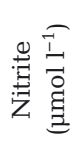 & 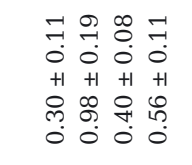 & 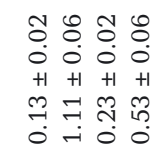 & 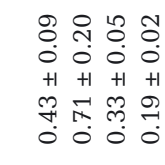 \\
\hline 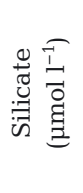 & 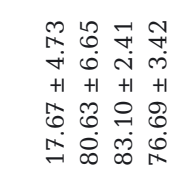 & 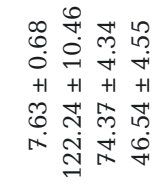 & 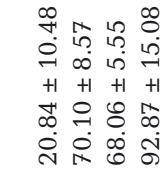 \\
\hline$\stackrel{\bigcirc}{\circ}$ & 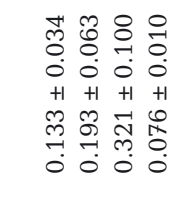 & $\left.\begin{array}{l}m \\
m \\
0 \\
0\end{array}\right)$ & 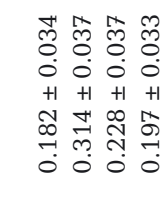 \\
\hline 贡 & 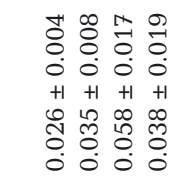 & 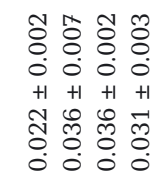 & 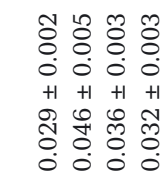 \\
\hline 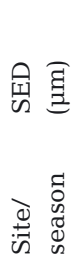 & 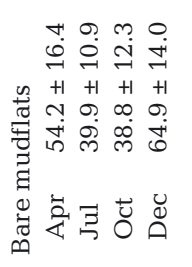 & 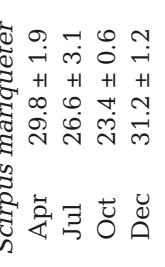 & 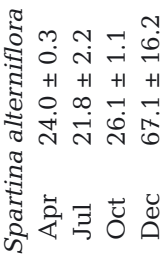 \\
\hline
\end{tabular}

along CAP1 (Fig. 3A). The ANOSIM test verified the pattern shown in CAP that there were significant differences among the 3 habitats $(\mathrm{R}=0.471$, $\mathrm{p}<0.01$; Table 2) and among the 4 seasons $(\mathrm{R}=$ 0.889, $\mathrm{p}<0.01$; Table 2). The RELATE procedure detected a significant cycle pattern within each habitat ( $\rho=0.475, p<0.01$ ). The vector overlays of the 12 environmental variables with the CAP axes is shown in Fig. 3C. Vectors for $\mathrm{pH}, \mathrm{SED}$, and nitrate point toward the bare mudflats and Scirpus marshes collected in winter, while silicate and vegetation height point to the sample clouds of the 2 meadows (Fig. 3C). All other nutrients as well as temperature and salinity point towards samples collected in summer (Fig. 3C).

\subsection{Community composition and variation partitioning}

A total of 60 ciliate species representing 42 genera, 30 families, 17 orders, and 9 classes were recorded (Table S2 \& S3). These included 26 species previously recorded in nearby salt marshes at Chongming Island (Xu et al. 2018); the other species were reported in salt marshes in Yangtze River Estuary for the first time. Generally, the most abundant species belonged to 2 classes, i.e. Litostomatea and Prostomatea (Table S3). Ciliate species composition clustered according to habitat and sampled season (Fig. 3B). For example, ciliate communities of Scirpus marshes are located in the negative range of CAP2, while that of Spartina marshes are located closer to communities of bare mudflats, which are in the positive range of CAP2 (Fig. 3B). Communities collected in October are separated from other seasons by CAP1 (Fig. 3B). ANOSIM demonstrated significant differences among the 3 habitats $(\mathrm{R}=0.198, \mathrm{p}<0.01$; Table 2) and among 4 seasons $(\mathrm{R}=0.471, \mathrm{p}<0.01$; Table 2$)$. The RELATE procedure detected a significant cycle pattern within each habitat $(\rho=0.177, p<0.01)$. Vector overlay of Pearson correlations of typical species with the CAP axes is shown in Fig. 3D. Vectors for 3 species (Holophrya caspica, H. aklitolophon, and Kentrophyllum setigerum) point toward the sample cloud for bare mudflats and Spartina marshes collected in October; vectors for 4 species (Litonotus sp1, $L$. fusidens, Metacystis striata, and Chaenea minor) point toward the samples cloud for Scirpus marshes collected in October and December; vectors for 3 species (Loxophyllum spirillum, Phialina salinarum, and Litonotus cygnus) point toward the samples cloud for bare mudflats and Spartina marshes col- 


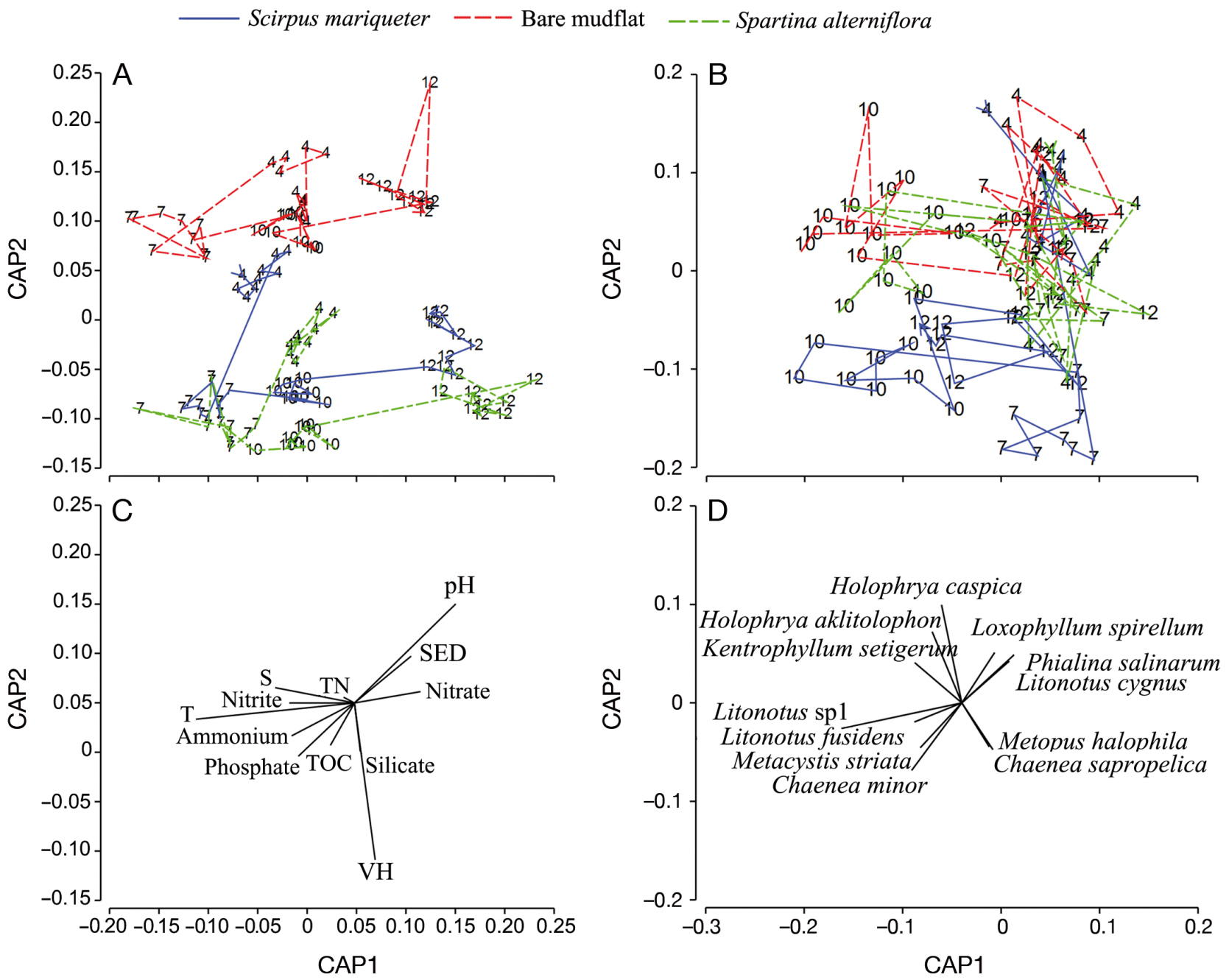

Fig. 3. (A,C) Canonical analysis of principal coordinates on Euclidean distance from normalized log-transformed environmental data and $(B, D)$ Bray-Curtis similarities from square-root-transformed benthic ciliate species abundance data, with (C) correlations of environmental variables, and (D) typical species (only vectors with correlation $>0.3$ are shown). The numbers along the plotted lines in (A) and (B) refer to sampling month. All replicate samples are included for analysis. S: salinity; SED: sediment grain size; T: temperature; TN: total nitrogen; TOC: total organic carbon

Table 2. Output from 3-way ANOSIM with 'sampling site' as a nested factor in 'habitat' but crossed with 'season', examining variance of environmental conditions, community composition, and 5 diversity indices between samples. AvTD: average taxonomic distinctness; $d$ : Margalef's richness; Env: environment variables; FDiv: functional divergence; N: total number of individuals; $S$ : total number of species. ${ }^{*} \mathrm{p}<0.05,{ }^{* *} \mathrm{p}<0.01$

\begin{tabular}{|c|c|c|c|c|c|c|c|c|}
\hline & Env & Species & Traits & $S$ & $\mathrm{~N}$ & $d$ & AvTD & FDiv \\
\hline Habitat & $0.399^{* *}$ & $0.198^{* *}$ & 0.126 & -0.053 & 0.111 & -0.043 & 0.033 & -0.03 \\
\hline Season & $0.882^{* *}$ & $0.471^{* *}$ & $0.291^{* *}$ & $0.204^{* *}$ & $0.286^{* *}$ & $0.131^{*}$ & $0.174^{* *}$ & 0.009 \\
\hline
\end{tabular}

lected in April, July, and December; and vectors for 2 species (Metopus halophila and Chaenea sapropelica) point toward the samples cloud for Scirpus marshes collected in April and July (Fig. 3B).

The results from RDA revealed that 6 environmental variables, i.e. SED, TOC, nitrite, phosphate, tem- perature, and height of vegetation, had significant influence on species composition by explaining 2.2, $1.9,1.0,2.2,1.1$, and $2.4 \%\left(\operatorname{adj} . \mathrm{R}^{2}\right)$ of total variation, respectively (Table 3 ).

Based on ANOSIM, ciliate trait composition shows significant differences among 4 seasons $(\mathrm{R}=$ 
0.291, $\mathrm{p}<0.01$; Table 2), but the difference among 3 habitats is not significant $(\mathrm{R}=0.126, \mathrm{p}>0.05$; Table 2). The results from RDA revealed that the trait composition was only related significantly to SED, nitrate, and temperature, which explain 2.8, 2.1 , and $2.3 \%$ (adj. $\mathrm{R}^{2}$ ) of the total variance, respectively (Table 3).

\subsection{Diversity patterns}

Based on ANOSIM, the differences among all 5 diversity indices among the 3 habitats are not significant (Fig. 4, Table 2).

According to the GLMs, $S$ showed a significant negative correlation with TOC and silicate, $\mathrm{N}$ did not

Table 3. Standardized coefficients for multiple regression (diversity indices), and adjusted $\mathrm{R}^{2}$ (\%) for redundancy analyses (RDA; community compositions). AIC: Akaike's information criterion; AvTD: average taxonomic distinctness; $d$ : Margalef's richness; FDiv: functional divergence; N: total number of individuals; $S$ : total number of species; SED: sediment grain size; T: temperature; TN: total nitrogen; TOC: total organic carbon; VH: height of vegetation. ${ }^{*} p<0.05,{ }^{* *} p<0.01$. $(-)$ : variables not selected by the forward selection before RDA or not included in the most parsimonious regression model based on AIC

\begin{tabular}{|c|c|c|c|c|c|c|c|}
\hline \multirow[t]{2}{*}{ Variable } & \multicolumn{2}{|c|}{$\longrightarrow$ RDA results -} & \multicolumn{5}{|c|}{ - Multiple regression results } \\
\hline & Species composition & Trait composition & $S$ & $\mathrm{~N}$ & $d$ & AvTD & FDiv \\
\hline SED & $2.2^{* *}$ & $2.8^{* *}$ & - & - & $0.10^{*}$ & - & - \\
\hline TN & - & - & - & - & - & - & $0.03^{*}$ \\
\hline TOC & $1.9^{* *}$ & - & $-0.23^{*}$ & -0.17 & - & - & - \\
\hline Silicate & - & - & $-0.21^{* *}$ & - & - & - & - \\
\hline Nitrite & $1.0^{*}$ & - & - & - & - & $0.16^{*}$ & -0.04 \\
\hline Ammonia & - & - & - & - & - & - & $-0.05^{* *}$ \\
\hline Phosphate & $2.2^{* *}$ & - & - & - & - & - & - \\
\hline Nitrate & - & $2.1^{*}$ & - & - & - & - & - \\
\hline $\mathrm{pH}$ & - & - & -0.15 & - & - & - & $-0.05^{*}$ \\
\hline Salinity & - & - & - & 0.21 & - & $0.32^{* *}$ & -0.03 \\
\hline $\mathrm{T}$ & $1.1^{* *}$ & $2.3^{* *}$ & - & - & $0.16^{* *}$ & - & - \\
\hline VH & $2.4^{* *}$ & - & - & -0.16 & - & - & -0.03 \\
\hline Intercept & & & $1.77^{* *}$ & $2.39^{* *}$ & $1.10^{* *}$ & $3.14^{* *}$ & $0.49^{* *}$ \\
\hline AIC & & & 234.48 & 332.95 & 83.637 & 212.57 & -100.6 \\
\hline
\end{tabular}
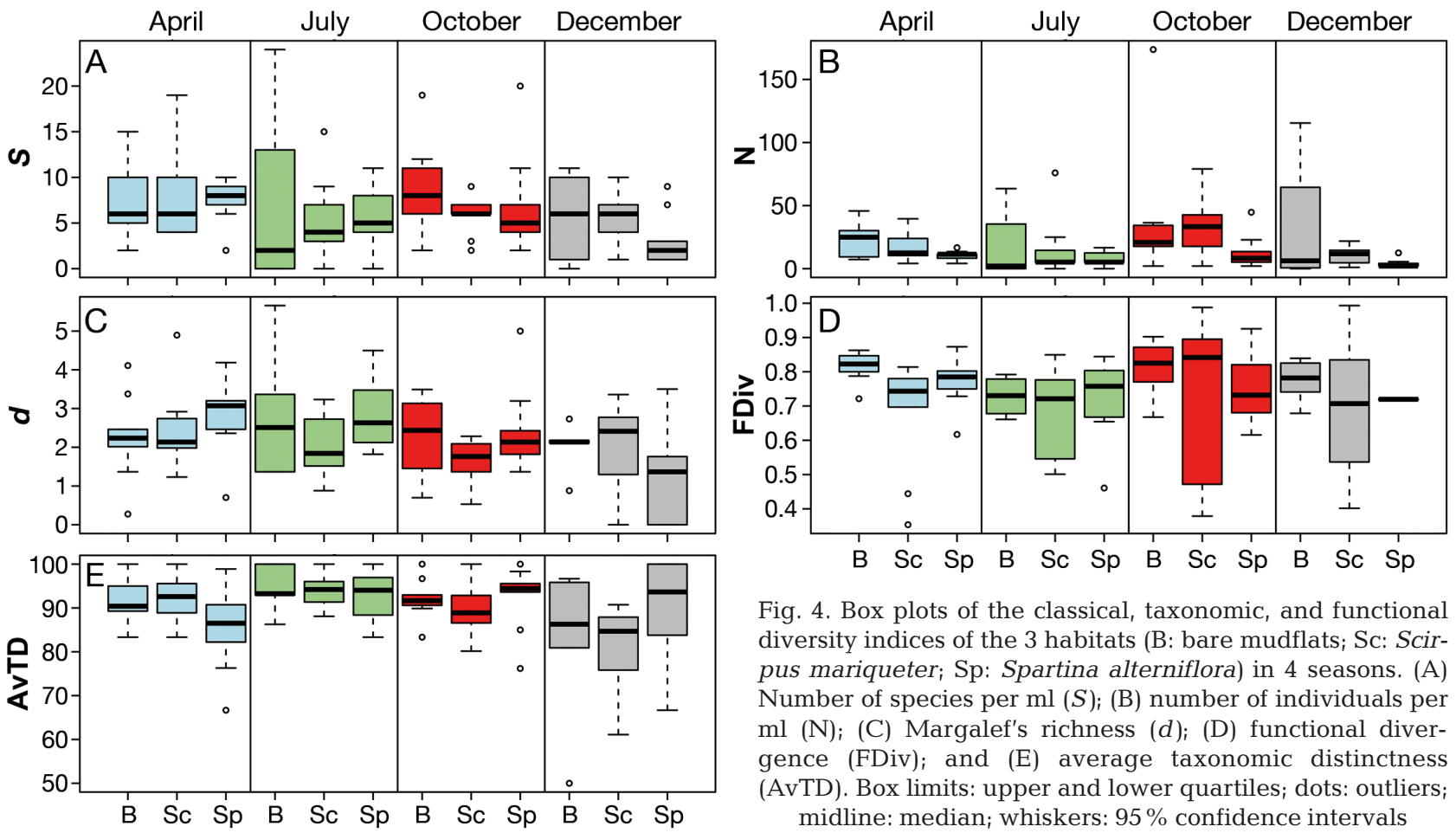

Fig. 4. Box plots of the classical, taxonomic, and functional diversity indices of the 3 habitats (B: bare mudflats; Sc: Scirpus mariqueter; Sp: Spartina alterniflora) in 4 seasons. (A) Number of species per ml $(S)_{\text {; }}$ (B) number of individuals per $\mathrm{ml}(\mathrm{N})$; (C) Margalef's richness $(d)$; (D) functional divergence (FDiv); and (E) average taxonomic distinctness (AvTD). Box limits: upper and lower quartiles; dots: outliers; midline: median; whiskers: $95 \%$ confidence intervals 
show significant correlation with any variables, $d$ scaled positively with SED and temperature, AvTD increased significantly with nitrite and salinity, and FDiv had a significantly positive relationship with TN, whereas the relationship with ammonia and $\mathrm{pH}$ was negative (Table 3).

\section{DISCUSSION}

\subsection{Effects on environmental condition}

It has been documented that changes in plant structure play a significant role in shaping the sedimentary abiotic condition (Talley \& Levin 2001). In the present study, we found significant differences in the environmental factors among the 3 habitats and 4 seasons. Among these variables, SED, TOC, and vegetation height showed clear differences among habitats, while others were more influenced by season (Fig. 2, Table 2). In our results, SED in bare mudflats is larger than in the 2 meadows. This finding is consistent with a previous study which found that plants trapped fine-grained sediment (Yang et al. 2008). We found comparatively higher TOC in Spartina stands than in the other 2 habitats. This result agrees with previous studies which noted that invasion of Spartina in the Yangtze River estuary has significant impacts on TOC in surface sediment, since this $\mathrm{C}_{4}$ plant species possesses a higher photosynthetic efficiency and greater biomass compared with $\mathrm{C}_{3}$ Scirpus (Cheng et al. 2006, Liao et al. 2007). The CAP analysis showed that the environmental variables of restored Scirpus stands are more similar to Spartina stands compared to bare mudflats. This suggests that the environmental conditions have been altered fundamentally due to the conversion of bare mudflats to Scirpus meadow in these areas and that the mere presence of vegetation has a stronger influence on shaping abiotic variables rather than the type of these 2 plants.

\subsection{Effects on microbenthic community structure}

In the present study, the species composition of the benthic ciliate community differed significantly among the 3 habitats. Previous studies on the influence of Spartina invasion on benthic community structure noted similar results for both meiobenthos and macrobenthos, whose communities changed significantly between invasive and native meadows in the Yangtze River estuary (Chen et al. 2007, Li et al.
2009). In contrast, in our study, the trait composition was not significantly different among the 3 habitats. These results were supported by RDA analysis showing that the vegetation height could explain $2.4 \%$ of the variation in species composition, while trait composition was only significant correlated with SED, nitrate, and temperature. Although SED varied among the 3 habitats, it also exhibited clear seasonal shifts, similar to nitrate and temperature, which may explain the significant difference in trait composition among the 4 seasons.

As with trait composition, none of the diversity indices of the ciliate communities were significantly different among the 3 habitats. Previous studies on the influences of Spartina spp. invasion on classic diversity of meio- and macrobenthic communities compared with uninvaded areas reached inconsistent conclusions. Chen et al. (2005) found lower numbers of individuals and richness of macrofauna in invaded meadows. Neira et al. (2005) reported species richness in Spartina stands to be higher than in native marshes but lower than in mudflats. Other studies showed non-significant differences in diversity between exotic and native marshes (Hedge \& Kriwoken 2000, Li et al. 2009, Wu et al. 2009, Quan et al. 2016) or changing impacts according to study sites and seasons (Talley \& Levin 2001). In the present study, although we found the species composition to be influenced significantly by the type of vegetation, the classic diversity indices did not change, which indicates that species replacement happened among habitats.

A previous study on meiobenthos postulated that altered species composition caused by exotic plant invasions may eventually impact the functioning of benthic ecosystems by altering the food web (Wu et al. 2009). However, Villéger et al. (2010) pointed out that changes in species composition due to species replacement do not necessarily lead to changes in ecosystem functions, since different species may share similar functions. The latter is supported by the present study: based on CAP analysis, the typical species in each habitat shifted, but most of them belong to Prostomatea and Litostomatea and share similar functional traits, which leads to unchanged AvTD and FDiv among habitats. Therefore, since typical species were replaced by taxonomically and functionally similar organisms, the effects of changes in vegetation type on benthic ecosystem functions might be small. Our findings agree with a previous study on the functional diversity of testate amoeba in a restored floodplain, which showed that the difference between the restored area and the reference site were not significant (Fournier et al. 2012). 
GLM analyses also showed that AvTD and FDiv strongly correlated with nutrients (TN, ammonia, and nitrite), as well as with $\mathrm{pH}$ and salinity, which indicates that nitrogen-related nutrients could have stronger effects on these 2 diversity indices. This finding is consistent with previous studies in that both taxonomic and functional diversity indices can be used to indicate anthropogenic impacts, such as nitrogen eutrophication (Xu et al. 2016, 2018). However, changes in the type of vegetation resulting from marsh restoration show little influence on these indices.

Other environmental variables such as hydrodynamic conditions could potentially be modified by Spartina invasion. Quan et al. (2016) found that, compared with Scirpus, stands of Spartina significantly decreased water velocity and increased sediment deposition rate, but macrofauna species density did not exhibit differences between habitats. We did not measure hydrodynamic conditions and did not detect significant differences in ciliate diversity among the 3 habitats, suggesting that hydrodynamic effects on benthic ciliates were not significant. A small amount of variation in ciliate species composition was explained $(10.8 \%)$ by the environmental variables in our study, which is generally smaller compared with benthic diatoms (21.6\% explained in Jyrkänkallio-Mikkola et al. 2017) and similar or larger than benthic bacteria (12.9\% explained in Heino et al. 2014, 3.4\% in Jyrkänkallio-Mikkola et al. 2017). Unexplained variation in ciliate communities in RDA may be related to some unmeasured variables, such as food supply or biological interactions (potential predators or competitors). Another shortcoming in the present study is that it was carried out for only $1 \mathrm{yr}$; longterm evaluation is required in future to study the changes in ciliate communities along a chronosequence following salt marsh restoration and compare the rate of colonization between microbenthos and macrobenthos.

\section{CONCLUSIONS}

Our results indicate that environmental variables among the 3 habitats differed significantly, especially SED, TOC, and vegetation height, which led to significantly different species composition of the benthic ciliate community. However, the difference in functional trait composition was not significant among the 3 habitats. Together with the results from the CAP analysis, we found that species replacement among the 3 habitats mainly occurred within 2 ciliate classes, i.e. Prostomatea and Litostomatea, sharing similar functional traits, and therefore led to unchanged taxonomic diversity and functional diversity. Our findings suggest that the effects of a change in vegetation due to Scirpus restoration on the benthic ciliate community were not significant at the time scale of the present study. Long-term monitoring is necessary in the future to study the temporal changes in ciliate communities following salt marsh restoration.

Acknowledgements. Y.X. was supported by the National Key R\&D Program of China (2017YFC0506001; 2016YFE 0133700) and K.S. was supported by the Royal Netherlands Institute of Sea Research, project no. PSA-SA-E-02. Both Y.X. and K.S. were supported by the project 'Coping with deltas in transition' within the Program of Strategic Scientific Alliances between China and the Netherlands (PSA).

\section{LITERATURE CITED}

Alphin TD, Posey MH (2000) Long-term trends in vegetation dominance and infaunal community composition in created marshes. Wetlands Ecol Manage 8:317-325

Anderson MJ, Gorley RN, Clarke KR (2008) PERMANOVA+ for PRIMER: guide to software and statistical methods. PRIMER-E, Plymouth

Barter M, Tonkinson D, Tang SX, Yuan X, Qian FW (1997) Wader numbers on Chongming Dao, Yangtze estuary, China, during early 1996 northward migration, and the conservation implications. Stilt 30:7-13

Bremner J, Rogers SI, Frid CLJ (2003) Assessing functional diversity in marine benthic ecosystems: a comparison of approaches. Mar Ecol Prog Ser 254:11-25

Carey PG (1992) Marine interstitial ciliates. Chapman \& Hall, London

Chen Z, Fu C, Wang H, Li B, Wu J, Chen J (2005) Effects of Spartina alterniflora invasions on the benthic macroinvertebrate community at Dongtan of Chongming salt marsh, the Yangtze River estuary. Wetland Sci 3:1-7

Chen H, Li B, Hu J, Chen J, Wu J (2007) Benthic nematode communities in the Yangtze River estuary as influenced by Spartina alterniflora invasions. Mar Ecol Prog Ser 336: 99-110

Cheng X, Luo Y, Chen J, Lin G, Chen J, Li B (2006) Shortterm $\mathrm{C}_{4}$ plant Spartina alterniflora invasions change the soil carbon in $\mathrm{C}_{3}$ plant-dominated tidal wetlands on a growing estuarine island. Soil Biol Biochem 38: 3380-3386

Clarke KR, Ainsworth M (1993) A method of linking multivariate community structure to environmental variables. Mar Ecol Prog Ser 92:205-219

Clarke KR, Gorley RN, Somerfield PJ, Warwick RM (2014) Change in marine communities: an approach to statistical analysis and interpretation, 3rd edn. PRIMER-E, Plymouth

* Cordier T, Lanzén A, Apothéloz-Perret-Gentil L, Stoeck T, Pawlowski J (2018) Embracing environmental genomics and machine learning for routine biomonitoring. Trends Microbiol 27:387-397 
Craft C, Sacco J (2003) Long-term succession of benthic infauna communities on constructed Spartina alterniflora marshes. Mar Ecol Prog Ser 257:45-58

Ferguson HJ, Rakocinski CF (2008) Tracking marsh restoration using macrobenthic metrics: implementing a functional approach. Wetlands Ecol Manage 16:277-289

Forster D, Filker S, Kochems R, Breiner HW, Cordier T, Pawlowski J, Stoeck T (2019) A comparison of different ciliate metabarcode genes as bioindicators for environmental impact assessments of salmon aquaculture. J Eukaryot Microbiol 66:294-308

Fournier B, Malysheva E, Mazei Y, Moretti M, Mitchell EA (2012) Toward the use of testate amoeba functional traits as indicator of floodplain restoration success. Eur J Soil Biol 49:85-91

Kan X, Cai Y, Choi C, Ma Z, Chen J, Li B (2009) Potential impacts of invasive Spartina alterniflora on spring bird communities at Chongming Dongtan, a Chinese wetland of international importance. Estuar Coast Shelf Sci 83: 211-218

Gedan KB, Silliman BR, Bertness MD (2009) Centuries of human-driven change in salt marsh ecosystems. Annu Rev Mar Sci 1:117-141

Gusmao JB, Brauko KM, Eriksson BK, Lana PC (2016) Functional diversity of macrobenthic assemblages decreased in response to sewage discharges. Ecol Indic 66:65-75

* Hamels I, Muylaert K, Sabbe K, Vyverman W (2005) Contrasting dynamics of ciliate communities in sandy and silty sediments of an estuarine intertidal flat. Eur $J$ Protistol 41:241-250

He Y, Li X, Guo W, Ma Z (2012) Division of labor in rhizomatous species: comparative performance of native and invasive species in the tidal marshes of the Yangtze River estuary, China. J Exp Mar Biol Ecol 422-423:122-128

* Hedge P, Kriwoken LK (2000) Evidence for effects of Spartina anglica invasion on benthic macrofauna in Little Swanport estuary, Tasmania. Austral Ecol 25:150-159

Heino J, Tolkkinen M, Pirttilä AM, Aisala H, Mykrä H (2014) Microbial diversity and community-environment relationships in boreal streams. J Biogeogr 41:2234-2244

Huspeni TC, Lafferty KD (2004) Using larval trematodes that parasitize snails to evaluate a saltmarsh restoration project. Ecol Appl 14:795-804

Jyrkänkallio Mikkola J, Meier S, Heino J, Laamanen T and others (2017) Disentangling multi scale environmental effects on stream microbial communities. J Biogeogr 44: $1512-1523$

Laliberté E, Legendre P, Shipley B (2014) FD: measuring functional diversity from multiple traits, and other tools for functional ecology. $\mathrm{R}$ package version 1.0-12. CRAN.R-project.org/package=FD

Lei Y, Xu K (2011) Techniques and operating procedures for the investigation of marine microbenthos. Oceanol Limnol Sin 1:157-164

* Lepš J, Bello F, Šmilauer P, Doležal J (2011) Community trait response to environment: disentangling species turnover vs intraspecific trait variability effects. Ecography 34 : 856-863

Li B, Liao C, Zhang X, Chen H and others (2009) Spartina alterniflora invasions in the Yangtze River estuary, China: an overview of current status and ecosystem effects. Ecol Eng 35:511-520

Li C, Tao Y, Zhao M, Yu K, Xu L, Fang S (2018) Soil characteristics and their potential thresholds associated with Scirpus mariqueter distribution on a reclaimed wetland coast. J Coast Conserv 22:1107-1116

* Li X, Bellerby R, Craft C, Widney SE (2018) Coastal wetland loss, consequences, and challenges for restoration. Anthropocene Coasts 1:1-15

* Liao C, Luo Y, Jiang L, Zhou X and others (2007) Invasion of Spartina alterniflora enhanced ecosystem carbon and nitrogen stocks in the Yangtze Estuary, China. Ecosystems 10:1351-1361

Lynn DH (2008) The ciliated protozoa: characterization, classification and guide to the literature, 3rd edn. Springer, Dordrecht

Lynn DH, Small EB (2002) Phylum Ciliophora Doflein, 1901. In: Lee JJ, Leedale GF, Bradbury PC (eds) An illustrated guide to the protozoa, 2nd edn. Society of Protozoologists, Lawrence, KS, p 371-656

Madoni P (2000) The acute toxicity of nickel to freshwater ciliates. Environ Pollut 109:53-59

McGill BJ, Enquist BJ, Weiher E, Westoby M (2006) Rebuilding community ecology from functional traits. Trends Ecol Evol 21:178-185

* Moseman SM, Levin LA, Currin C, Forder C (2004) Colonization, succession, and nutrition of macrobenthic assemblages in a restored wetland at Tijuana Estuary, California. Estuar Coast Shelf Sci 60:755-770

Neira C, Levin LA, Grosholz ED (2005) Benthic macrofaunal communities of three sites in San Francisco Bay invaded by hybrid Spartina, with comparison to uninvaded habitats. Mar Ecol Prog Ser 292:111-126

* Pratt JR, Cairns J (1985) Functional groups in the protozoa: roles in differing ecosystems. J Protozool 32:415-423

* Quan W, Zhang H, Wu Z, Jin S, Tang F, Dong J (2016) Does invasion of Spartina alterniflora alter microhabitats and benthic communities of salt marshes in Yangtze River estuary? Ecol Eng 88:153-164

R Core Team (2018) R: a language and environment for statistical computing, version 3.5.3. R Foundation for Statistical Computing, Vienna. www.r-project.org

Stoeck T, Kochems R, Forster D, Lejzerowicz F, Pawlowski J (2018) Metabarcoding of benthic ciliate communities shows high potential for environmental monitoring in salmon aquaculture. Ecol Indic 85:153-164

* Talley TS, Levin LA (2001) Modification of sediments and macrofauna by an invasive marsh plant. Biol Invasions 3: 51-68

*Villéger S, Mason NWH, Mouillot D (2008) New multidimensional functional diversity indices for a multifaceted framework in functional ecology. Ecology 89:2290-2301

* Villéger S, Miranda JR, Hernández DF, Mouillot D (2010) Contrasting changes in taxonomic vs. functional diversity of tropical fish communities after habitat degradation. Ecol Appl 20:1512-1522

Villéger S, Novack-Gottshall PM, Mouillot D (2011) The multidimensionality of the niche reveals functional diversity changes in benthic marine biotas across geological time. Ecol Lett 14:561-568

*Wang R, Yuan L, Zhang L (2010) Impacts of Spartina alterniflora invasion on the benthic communities of salt marshes in the Yangtze Estuary, China. Ecol Eng 36:799-806

*Warwick RM, Clarke KR (1995) New biodiversity measures reveal a decrease in taxonomic distinctness with increasing stress. Mar Ecol Prog Ser 129:301-305

Wheisse T (2006) Freshwater ciliates as ecophysiological model organisms-lessons from Daphnia, major achievements, and future perspectives. Arch Hydrobiol 167: $371-402$ 
Weisse T, Sonntag B (2016) Ciliates in planktonic food webs: communication and adaptive response. In: Witzany G, Nowacki M (eds) Biocommunication of ciliates. Springer, Cham, p 351-372

Whitcraft CR, Levin LA (2007) Regulation of benthic algal and animal communities by salt marsh plants: impact of shading. Ecology 88:904-917

Wu Y, Wang C, Zhang X, Zhao B, Jiang L, Chen J, Li B (2009) Effects of saltmarsh invasion by Spartina alterniflora on arthropod community structure and diets. Biol Invasions 11:635-649

Xu Y, Soininen J (2019) Spatial patterns of functional diversity and composition in marine benthic ciliates along the coast of China. Mar Ecol Prog Ser 627:49-60

Xu K, Du Y, Lei Y, Dai R (2010) A practical method of Ludox

Editorial responsibility: Robinson Fulweiler,

Boston, Massachusetts, USA density gradient centrifugation combined with protargol staining for extracting and estimating ciliates in marine sediments. Eur J Protistol 46:263-270

* Xu H, Jiang Y, Xu G (2016) Identifying functional species pool of planktonic protozoa for discriminating water quality status in marine ecosystems. Ecol Indic 62: 306-311

Xu Y, Stoeck T, Forster D, Ma Z, Zhang L, Fan X (2018) Environmental status assessment using biological traits analyses and functional diversity indices of benthic ciliate communities. Mar Pollut Bull 131:646-654

* Yang S, Li H, Ysebaert T, Bouma T and others (2008) Spatial and temporal variations in sediment grain size in tidal wetlands, Yangtze Delta: on the role of physical and biotic controls. Estuar Coast Shelf Sci 77:657-671

Submitted: August 28, 2019; Accepted: January 20, 2020 Proofs received from author(s): March 6, 2020 